\title{
Análise do efeito do uso a longo prazo de benzodiazepínicos por idosos: uma revisão sistemática de literatura
}

\author{
Analysis of the effect of long-term use of benzodiazepines by the elderly: a systematic literature \\ review
}

Análisis del efecto del uso prolongado de benzodiazepinas en ancianos: revisión sistemática de la literatura

\author{
Ana Gabriela Antunes Cardoso \\ ORCID: https://orcid.org/0000-0002-3474-8178 \\ Centro Universitário de Patos de Minas, Brasil \\ E-mail: anagabriela.acg@gmail.com \\ Luciano Rezende dos Santos \\ ORCID: https://orcid.org/0000-0002-2952-8218 \\ Centro Universitário de Patos de Minas, Brasil \\ E-mail: lucianors79@gmail.com \\ Amanda de Fátima Souza \\ ORCID: https://orcid.org/0000-0002-5236-5787 \\ Centro Universitário de Patos de Minas, Brasil \\ E-mail: amandafatima@unipam.edu.br \\ Bárbara Queiroz de Figueiredo \\ ORCID: https://orcid.org/0000-0003-1630-4597 \\ Centro Universitário de Patos de Minas, Brasil \\ E-mail: barbarafigueiredo@unipam.edu.br \\ Eduarda Canedo Nogueira \\ ORCID: https://orcid.org/0000-0002-3654-9228 \\ Centro Universitário de Patos de Minas, Brasil \\ E-mail: eduardacanedo@ hotmail.com \\ Emilayne Nicácio Dias Brito \\ ORCID: https://orcid.org/0000-0002-4002-8847 \\ Centro Universitário de Patos de Minas, Brasil \\ E-mail: emilaynendb@unipam.edu.br \\ Giselly Nunes Silva \\ ORCID: https://orcid.org/0000-0001-6471-9274 \\ Centro Universitário de Patos de Minas, Brasil \\ E-mail: gisellynunes@ unipam.edu.br \\ Rafaela Alves Fernandes \\ ORCID: https://orcid.org/0000-0002-0050-0844 \\ Centro Universitário de Patos de Minas, Brasil \\ E-mail: rafaelaaf@unipam.edu.br
}

\begin{abstract}
Resumo
Introdução: Os benzodiazepínicos são os psicofármacos mais utilizados globalmente e possuem propriedades farmacológicas que propiciam ação sedativa, hipnótica, ansiolítica, anticonvulsivante e relaxante muscular. A eficácia dos benzodiazepínicos é bem documentada nos tratamentos de curta duração, porém o uso prolongado é contraindicado devido aos riscos de efeitos adversos, incluindo a dependência.Objetivo: evidenciar o efeito a longo prazo do uso de benzodiazepínicos por idosos. Metodologia: Foi feita uma revisão bibliográfica e webliográfica, realizada no período de julho de 2020 a agosto de 2021, onde foram utilizadas as bases de dados Scientific Electronic Library Online - SciELO, US National Library of Medicine Nacional Institutes of Health (PubMed), Biblioteca Virtual em Saúde (BVS) e Google Acadêmico. Foi utilizada também a consulta a livros e periódicos presentes na Biblioteca do Centro Universitário de Patos de Minas. Após aplicados os critérios de inclusão e de exclusão foi realizada análise de 22 publicações. Resultados e Discussão: O estudo das bibliografias selecionadas mostrou que o uso de benzodiazepínicos entre idosos tem alta prevalência. Além disso, essa classe de medicamentos não é recomendada para uso por longevos, agravando suas consequências ainda mais quando o tratamento é realizado a longo prazo, exacerbando uma série de eventos adversos sem apresentar eficácia nesse formato de uso. Conclusão: é importante buscar formas menos prejudiciais para sanar as queixas em relação a sono e ansiedade em longevos e também capacitar os profissionais de saúde, a fim de identificar medicamentos com uso não recomendado para idosos e propor formas de otimizar as prescrições.
\end{abstract}


Palavras-chave: Benzodiazepínicos; Saúde do Idoso; Envelhecimento; Uso crônico.

\begin{abstract}
Introduction: Benzodiazepines are the most used psychotropic drugs globally and have pharmacological properties that provide sedative, hypnotic, anxiolytic, anticonvulsant and muscle relaxant action. The effectiveness of benzodiazepines is well documented in short-term treatments, but long-term use is contraindicated due to the risk of adverse effects, including dependence. Purpose: to demonstrate the long-term effect of benzodiazepine use by the elderly. Methodology: A bibliographic and webliographic review was carried out from July 2020 to August 2021, using the Scientific Electronic Library Online - SciELO, US National Library of Medicine, National Institutes of Health (PubMed), Library databases. Virtual Health (BVS) and Google Scholar. It was also used the consultation of books and periodicals present in the Library of the University Center of Patos de Minas. After applying the inclusion and exclusion criteria, an analysis of 22 publications was performed. Results and Discussion: The study of selected bibliographies showed that the use of benzodiazepines among the elderly has a high prevalence. In addition, this class of drugs is not recommended for use by the oldest old, aggravating its consequences even more when the treatment is carried out in the long term, exacerbating a series of adverse events without being effective in this form of use. Conclusion: it is important to look for less harmful ways to remedy complaints about sleep and anxiety in the oldest old and also to train health professionals in order to identify medications not recommended for the elderly and propose ways to optimize prescriptions.
\end{abstract}

Keywords: Benzodiazepines; Elderly Health; Aging; Chronic use.

\begin{abstract}
Resumen
Introducción: Las benzodiazepinas son los fármacos psicotrópicos más utilizados a nivel mundial y poseen propiedades farmacológicas que aportan acción sedante, hipnótica, ansiolítica, anticonvulsivante y relajante muscular. La eficacia de las benzodiazepinas está bien documentada en tratamientos a corto plazo, pero el uso a largo plazo está contraindicado debido al riesgo de efectos adversos, incluida la dependencia. Objetivo: demostrar el efecto a largo plazo del uso de benzodiazepinas en personas de edad avanzada. Metodología: Se realizó una revisión bibliográfica y webliográfica de julio de 2020 a agosto de 2021, utilizando la Scientific Electronic Library Online SciELO, la Biblioteca Nacional de Medicina de EE. UU., Los Institutos Nacionales de Salud (PubMed), las bases de datos de la Biblioteca. Virtual Health (BVS) y Google. Erudito. También se aprovechó la consulta de libros y publicaciones periódicas presentes en la Biblioteca del Centro Universitario de Patos de Minas. Tras aplicar los criterios de inclusión y exclusión, se realizó un análisis de 22 publicaciones. Resultados y Discusión: El estudio de bibliografías seleccionadas mostró que el uso de benzodiazepinas entre los ancianos tiene una alta prevalencia. Además, esta clase de fármacos no es recomendable para su uso por los ancianos, agravando aún más sus consecuencias cuando el tratamiento se lleva a cabo a largo plazo, exacerbando una serie de eventos adversos sin ser efectivos en este formato de uso. Conclusión: es importante buscar formas menos dañinas de remediar las quejas sobre el sueño y la ansiedad en los ancianos y también capacitar a los profesionales de la salud para identificar los medicamentos no recomendados para los ancianos y proponer formas de optimizar las prescripciones.
\end{abstract}

Palabras clave: Benzodiazepinas; Salud de los ancianos; Envejecimiento; Uso crónico.

\title{
1. Introdução
}

Os benzodiazepínicos são os psicofármacos mais utilizados globalmente e possuem propriedades farmacológicas que propiciam ação sedativa, hipnótica, ansiolítica, anticonvulsivante e relaxante muscular (Oliveira et al. 2020). A eficácia dos benzodiazepínicos é bem documentada nos tratamentos de curta duração, porém o uso prolongado é contraindicado devido aos riscos de efeitos adversos, incluindo a dependência (Firmino et al. 2011). Alguns estudos mostram discreta redução recente no padrão de prescrição de benzodiazepínicos e que o grupo que faz uso mais frequentemente é o de idosos (AMB, 2013). Admite-se que o consumo de benzodiazepínicos por idosos está relacionado ao fato de o envelhecimento ser acompanhado pelo aparecimento de transtornos do sono, depressão e das doenças neurológicas degenerativas (Fegadolli et al. 2019). No Brasil, pessoas idosas são consumidoras frequentes de benzodiazepínicos, como evidenciam estudos epidemiológicos desenvolvidos em diferentes populações, sejam elas residentes em comunidade, com prevalência estimada de $22 \%$, ou usuárias de serviços de saúde, cuja prevalência chega a 30\% (Alvarenga et al. 2015).

A maior utilização de benzodiazepínicos em pacientes com faixa etária mais elevada apresenta-se inadequada, pois, sabe-se que esses fármacos, em especial aqueles de meia vida longa não são recomendados para idosos, já que produzem sedação prolongada aumentando o risco de quedas e, consequentemente, de fraturas do quadril. Além 
disso, nos idosos, o risco pode ser potencializado devido às alterações fisiológicas, que interferem na farmacocinética e na farmacodinâmica dos medicamentos, tornando esta população mais vulnerável às interações medicamentosas e reações adversas. Quando há a necessidade de utilização destes medicamentos em pacientes idosos, os benzodiazepínicos recomendados são aqueles de ação intermediária ou curta, mesmo assim, em doses mais baixas (Netto et al. 2012). Diante disso, esse estudo teve como objetivo realizar, por meio de uma revisão literária, uma análise do uso de benzodiazepínicos por idosos, analisando aspectos epidemiológicos; indicações, uso inapropriado, eventos adversos e manejo adequado.

\section{Metodologia}

Foi realizada uma revisão literária, por meio de consulta a livros e periódicos presentes na Biblioteca do Centro Universitário de Patos de Minas e a artigos científicos selecionados através de busca em base de dados, no período de julho de 2020 a agosto de 2021. As seguintes bases de dados sistemáticas foram utilizadas nas buscas: Scientific Electronic Library Online - SciELO, US National Library of Medicine Nacional Institutes of Health (PubMed), Biblioteca Virtual em Saúde (BVS) e Google Acadêmico.

Foram incluídos os artigos e livros que abordavam características dos benzodiazepínicos, das alterações fisiológicas e resposta de idosos a medicações, e a relação entre o uso de benzodiazepínicos a longo prazo e seus efeitos nos idosos, escritos em inglês e português, publicados entre 2009 e 2021. Os critérios de exclusão foram estudos do tipo relato de caso e monografias e artigos que não responderam à questão do estudo. Após leitura de todo o material, os critérios de inclusão e exclusão possibilitaram a análise de 22 publicações. Foram usadas as seguintes palavras-chave e suas combinações, em inglês e português: benzodiazepínicos; idoso; saúde do idoso; polifarmácia; psicofármacos; envelhecimento; medicamentos inapropriados.

\section{Resultados}

\subsection{Benzodiazepínicos}

\subsubsection{Conceitos}

Os benzodiazepínicos, que devem seu nome à sua estrutura molecular, constituída por um anel benzeno, foram sintetizados por Leo H. Sternbach na metade do século passado, que observou um efeito anticonvulsivante e de controle da agressividade em animais de laboratório. Foi classificado como uma substância de baixa toxicidade e, portanto, segura. A empolgação inicial com a descoberta deu lugar à preocupação quando os primeiros pesquisadores alertaram, no final de década de 70, para os riscos de dependência, sintomas de abstinência e potencial abuso (AMB, 2013).

Os benzodiazepínicos são responsáveis por cerca de 50\% de toda a prescrição de psicotrópicos (AMB, 2013). Constituem o grupo mais importante e de maior uso prático dos hipnóticos, seguramente graças às suas quatro atividades fundamentais: ansiolítica, hipnótica, anticonvulsivante e relaxante muscular, além de, eventualmente, um efeito amnésico (Silva, 2017).

\subsubsection{Classificação Química}

Os benzodiazepínicos são agentes sedativos-hipnóticos amplamente utilizados. Todas as estruturas consistem em 1,4benzodiazepínicos, e a maioria contém um grupo carboxamida na estrutura em anel heterocíclica de sete membros. É necessário um substituinte na posição 7, como um halogênico ou grupo nitro, para a atividade sedativo-hipnótica (Katzung et al. 2017). 
Quadro 1: Benzodiazepínicos disponíveis no Brasil

\begin{tabular}{|c|c|}
\hline Nome Químico & Nome Comercial \\
\hline Alprazolam & Apraz, Frontal, Tranquinal, Altrox \\
\hline Bromazepam & Lexotam, Deptran, Somalium, Sulpam \\
\hline Buspirona & Ansitec, Bromoprim, Buspanil, Buspa \\
\hline Clobazam & Frizium, Urbanil \\
\hline Clonazepam & Rivotril, Clonotril \\
\hline Clordiazepóxido & Psicosedim \\
\hline Cloxazolam & Olcadil, Elum \\
\hline Diazepam & Diazepam, Noam, Valium, Ansilive \\
\hline Lorazepam & Lorax, Lorium, Mesmerim \\
\hline
\end{tabular}

Fonte: Leonardi et al. (2017).

\subsubsection{Mecanismo de Ação}

Do ponto de vista neurofisiológico, os benzodiazepínicos deprimem a atividade elétrica na formação reticular, o que explica o efeito hipnótico sedativo e a diminuição do nível de alerta cortical. De modo mais eficaz, essas substâncias deprimem o sistema límbico, notadamente o núcleo amigdaloide, o que explicaria o efeito ansiolítico e a ação antagonista dessas drogas para as convulsões produzidas pelos anestésicos locais, uma vez que se sabe que essas convulsões são determinadas por ativação da amígdala e do hipocampo (Silva, 2017).

Ao nível celular, o que ocorre é que os benzodiazepínicos atuam fazendo parte de um complexo existente nas membranas celulares dos neurônios, envolvendo o receptor dos benzodiazepínicos, o receptor GABA, alguns peptídeos com função moduladora e o canal de cloro. Dessa forma, os benzodiazepínicos facilitam a transmissão inibitória do sistema nervoso central (Silva, 2017).

Existem três tipos principais de receptores de GABA e numerosos subtipos. Os principais tipos são os receptores de $\mathrm{GABA}_{\mathrm{A}}, \mathrm{GABA}_{\mathrm{B}}$ e $\mathrm{GABA}_{\mathrm{C}}$. Os receptores de $\mathrm{GABA}_{\mathrm{A}}$ e $\mathrm{GABA}_{\mathrm{C}}$ são canais iônicos controlados por ligantes e fazem parte de um complexo macromolecular que forma um canal de cloreto inibitório (Stahl, 2013). Os benzodiazepínicos são fármacos de alta afinidade e altamente seletivos, que se ligam a um único sítio dos receptores $\mathrm{GABA}_{\mathrm{A}}$ contendo as subunidades $\alpha 1, \alpha 2, \alpha 3$ ou $\alpha 5$ e uma subunidade $\gamma$. Atuam como moduladores alostéricos positivos, potencializando a regulação dos canais dos receptores $\mathrm{GABA}_{\mathrm{A}}$ na presença de GABA. Aumentam a frequência de abertura dos canais na presença de baixas concentrações de GABA, e, em concentrações de GABA semelhantes às observadas nas sinapses, a desativação do receptor é prolongada. Além disso, os receptores $\mathrm{GABA}_{\mathrm{A}}$ no estado aberto apresentam maior afinidade pelo GABA que no estado fechado, de modo que a capacidade dos benzodiazepínicos de favorecer a abertura do canal resulta, secundariamente, em uma afinidade agonista aparentemente maior (Golan et al. 2012).

\subsubsection{Farmacocinética}

Os benzodiazepínicos, na sua maioria, são absorvidos em 1 a 3 horas, quando usados oralmente. Alguns podem ser usados parenteralmente, como o Diazepam, o Flunitrazepam, o Lorazepam e o Midazolam. Em geral, a absorção da droga, quando usada por via intramuscular, é irregular, salvo com o Lorazepam, e as substâncias lipossolúveis são mais bem absorvidas que as hidrossolúveis. Quanto ao uso intravenoso, deve-se alertar para a irritação venosa com dor à injeção, flebite e trombose que podem ocorrer com alguns benzodiazepínicos lipossolúveis, como o Diazepam, reações essas inexistentes com preparados hidrossolúveis, como o Midazolam (Silva, 2017).

Os benzodiazepínicos se ligam, em percentual elevado, às proteínas plasmáticas, principalmente à albumina (Silva, 2017). Em estudos moleculares, foi constatado que a potência desses fármacos se correlaciona com sua hidrofobia. Todavia, eles se ligam em grandes proporções às proteínas plasmáticas, como a albumina, e a hidrofobia aumenta a ligação às proteínas, 
e reduz, portanto, a concentração livre e o transporte do fármaco pela barreira hematoencefálica. Além disso, em determinados estados clínicos associados a baixos níveis de albumina, como na hemodiluição aguda ou disfunção hepática, pode-se observar um notável aumento na potência clínica das benzodiazepinas (Golan et al. 2012).

A biotransformação de alguns benzodiazepínicos em metabólitos ativos pode ser importante na escolha da droga; o Clordiazepóxido, o Diazepam e o Flurazepam são transformados em metabólitos ativos, com meia-vida de eliminação longa, enquanto o Lorazepam, o Oxazepam e o Triazolam não formam metabólitos ativos de longa duração, o mesmo ocorrendo com o Midazolam (Silva, 2017).

O metabolismo hepático é responsável pela depuração de todos os benzodiazepínicos. Os padrões e as taxas de metabolismo dependem de cada fármaco em particular. A maioria dos benzodiazepínicos sofre oxidação microssômica, incluindo N-desalquilação e hidroxilação alifática catalisada por isozimas do citocromo P450, especialmente a CYP3A4. Subsequentemente, os metabólitos são conjugados para formar glicuronídeos, que são excretados na urina. Todavia, muitos metabólitos de fase de oxidação dos benzodiazepínicos são farmacologicamente ativos, alguns com meias-vidas longas (Katzung et al. 2017).

\subsubsection{Aplicações Clínicas}

Segundo Golan et al. (2012), os benzodiazepínicos são utilizados como potencializadores do sono, ansiolíticos, sedativos, antiepilépticos e relaxantes musculares, bem como para tratamento dos sintomas de abstinência do etanol.

Em situações de cuidados agudos, como na preparação do paciente para procedimentos invasivos, o midazolam é frequentemente usado como ansiolítico/sedativo/amnésico de início rápido e ação curta. Os benzodiazepínicos são frequentemente usados como sedativos para procedimentos desconfortáveis e de curta duração associados a dor aguda mínima, como a endoscopia. Quando administradas antes de anestesia geral, os benzodiazepínicos reduzem a necessidade de agentes hipnóticos (Golan et al. 2012).

\subsubsection{Reações Adversas}

Após o uso oral, pode ocorrer no dia seguinte, ou até algumas horas depois, sedação, acompanhada de sensação de fadiga e, eventualmente, vertigens, reações essas dependentes da dose, que são, entretanto, mais comuns em pacientes idosos e debilitados. Reações mais graves como depressão respiratória, apneia, depressão miocárdica com hipotensão grave são difíceis de ocorrer nas doses terapêuticas, aparecendo apenas na superdosagem, principalmente em pacientes idosos ou quando em associação com outros depressores do sistema nervoso central, como morfinomiméticos, barbitúricos e bebidas alcoólicas (Silva, 2017). Problemas de atenção e memória podem ocorrer mesmo com o uso agudo, mas são bastante frequentes com o uso crônico (AMB, 2013).

\subsubsection{Dependência e Tolerância}

São complicações do uso de benzodiazepínicos a tolerância, a dependência e a síndrome de abstinência (AMB, 2013). A dependência física e psíquica com o uso de benzodiazepínicos, apesar de não ser tão frequente como se observa com os barbitúricos e morfínicos, pode, entretanto, desenvolver-se de forma muito lenta, principalmente quando esse uso é feito em associação com outras drogas de forma regular e por tempo prolongado (Silva, 2017). O desenvolvimento da dependência relaciona-se com o tempo de uso, além de fatores individuais (AMB, 2013).

Discreta tolerância pode ocorrer, levando à necessidade de pequeno aumento nas doses iniciais. Alguns autores falam ainda na possibilidade de tolerância cruzada para com os hipnóticos não benzodiazepínicos, como barbituratos, metaqualona e até álcoois (Silva, 2017). O tempo para desenvolvimento de tolerância aos benzodiazepínicos varia 
enormemente de indivíduo para indivíduo, mas em geral a tolerância para os efeitos hipnóticos e sedativos aparece mais precocemente do que a tolerância para os efeitos ansiolíticos e cognitivos (AMB, 2013). Dado o potencial de desenvolvimento de tolerância, dependência e adição, seu uso deve ser intermitente (Golan et al. 2012).

\subsubsection{Uso de benzodiazepínicos a longo prazo}

Algumas complicações são raras, ocorrendo com maior frequência após uso prolongado e podem incluir disfunção sexual, ganho de peso, alterações de ciclo menstrual, erupções cutâneas, anomalias hematológicas e agravamento de glaucoma de ângulo fechado. Em 1\% dos casos, pode ocorrer a reação paradoxal, com instabilidade emocional, agitação psicomotora, irritabilidade e confusão mental. Reações paradoxais são mais frequentes com o uso concomitante de álcool (AMB, 2013). Os benzodiazepínicos cujo fármaco original ou cujos metabólitos ativos apresentam meias-vidas longas têm mais tendência a causar efeitos cumulativos com a administração de múltiplas doses (Katzung et al. 2017).

\subsection{Saúde do idoso}

\subsubsection{Conceitos}

O conceito de saúde do idoso está fortemente ancorado na "capacidade individual de satisfação de suas necessidades biopsicossociais, independentemente da idade ou da presença de doenças”. Essa capacidade funcional resulta da combinação entre fatores intrínsecos - sua capacidade física e mental - e extrínsecos, levando a uma marcante e progressiva heterogeneidade entre os indivíduos idosos ao longo do processo de envelhecimento (SBIBAE, 2019).

\subsubsection{Dados Epidemiológicos}

No Brasil, estima-se que a população com 60 anos ou mais seja de 30 milhões de pessoas, representando cerca de 14\% da população geral (210 milhões habitantes). É o segmento populacional com maior taxa de crescimento - acima de $4 \%$ ao ano -, passando de 14,2 milhões, em 2000, para 19,6 milhões, em 2010, e devendo atingir 41,5 milhões, em 2030, e 73,5 milhões, em 2060 (SBIBAE, 2019). Desta forma, um maior contingente de idosos consegue alcançar idades mais avançadas, próximas ao limite da expectativa de vida da espécie (Freitas, 2018).

Um estudo de base populacional evidenciou que cerca de um em cada dez idosos do município de São Paulo consumiu psicotrópicos, com destaque para os antidepressivos e benzodiazepínicos, alguns considerados inapropriados segundo os critérios de Beers. No que tange ao risco, mulheres e idosos submetidos à polifarmácia merecem atenção diferenciada quanto ao tipo de psicotrópico, ajuste posológico e tempo de tratamento, com vistas à minimização dos desfechos adversos a que estão sujeitos (Ramos et al. 2020).

Os idosos de hoje, apesar de terem escapado das causas de morte mais comuns, desenvolveram condições de baixa letalidade que geram significativo comprometimento da qualidade de vida. Um terço a cada ano sofre uma queda, $11 \%$ das quais originam lesões graves de tecidos moles ou fraturas, inclusive do fêmur, agravando os problemas de mobilidade, comprometendo a independência e provocando outras quedas. Outros sofrem de depressão, ansiedade e distúrbios do sono, cujo reconhecimento e manejo é mais difícil nesta idade, e utilizam medicamentos psicoativos inadequados, que aumentam o risco de quedas (Freitas, 2018). Um idoso pode usar simultaneamente de duas a seis prescrições médicas e ainda praticar automedicação com mais de dois medicamentos (Ramos et al. 2020).

\subsubsection{Alterações Fisiológicas}

A senescência é o processo normal de envelhecimento que promove alterações das funções orgânicas (Oliveira, et al., 2017). O envelhecimento fisiológico reduz a vitalidade do indivíduo, definida como capacidade de defesa contra as agressões 
dos meios interno e externo, gerando maior vulnerabilidade dessa população. Essa redução fisiológica da vitalidade atribuída ao envelhecimento normal, por si só, é insuficiente para gerar incapacidades ou dependência funcional. Por sua vez, o envelhecimento patológico, consequência de uma ou mais doenças, e agravado por barreiras relacionadas aos fatores ambientais e contextuais, compromete, de forma mais intensa, a vitalidade do indivíduo e está associado ao declínio funcional (SBIBAE, 2019).

Segundo Freitas (2018), há controvérsias em relação ao início do envelhecimento, porém, por volta dos 25 anos já podemos observar modificações na composição corporal. Toda a celularidade diminui, reduzindo a função dos órgãos, continuamente. Ocorre diminuição da água intracelular, tornando o organismo da pessoa idosa desidratado, fisiologicamente. Daí devemos ficar alertas ao prescrevermos fármacos hidrossolúveis pois elas estarão em maior concentração, podendo ocorrer efeitos indesejáveis. Aqui também teremos que dar atenção às regras farmacológicas, pois as substâncias lipossolúveis, como as de ação central, terão seu tempo de ação aumentado. A redução da água corporal e da produção de albumina, associada ao aumento do tecido adiposo, impacta na fase de distribuição de substâncias (Oliveira et al. 2017).

A pele se torna seca, por diminuição das glândulas sebáceas, e espessada, com as papilas dérmicas menos profundas, levando a menor junção entre a epiderme e a derme, facilitando a formação de bolhas e predispondo a lesões. A administração de medicamentos pela via transdérmica possibilita a aplicação de agentes terapêuticos de forma indolor e com poucos efeitos adversos. (Freitas, 2018).

As alterações musculoesqueléticas, diferentemente das alterações cutâneas, geram dor e algum grau de dificuldade de locomoção (Ribeiro et al., 2009). A massa muscular diminui quase $50 \%$ entre os 20 e 90 anos, e a força muscular, que é máxima por volta dos 30 anos, sofre perda de $15 \%$ por década a partir dos 50 anos. Essa perda é mais acelerada, chegando a 30\%, por década, aos 70 anos e, praticamente a metade aos 80 anos. (Freitas, 2018). Há, ainda, redução da densidade óssea e diminuição da força muscular e da massa magra, enquanto ocorre aumento da massa gorda (Oliveira et al. 2017).

No sistema cardiovascular, o miocárdio sofre progressiva degeneração de fibras musculares, reduzindo sua complacência e frequência (Oliveira et al. 2017). A diminuição da capacidade contrátil causa aumento do coração que esconde a atrofia das células contráteis. Em uma aparente contradição, as câmaras cardíacas dilatadas e o coração senil, embora atrófico em número celular, morfologicamente, é hipertrófico (Freitas, 2018).

O envelhecimento leva gradualmente a um declínio da memória, atualmente chamado de comprometimento cognitivo leve. Uma das principais causas da deterioração cognitiva com o passar da idade é a diminuição neuronal e de neurotransmissores, cujo grau varia nas diferentes partes do cérebro (Ribeiro et al. 2009). Além das modificações do sistema nervoso central, há também mudanças dos nervos periféricos e da musculatura. A consequência dessas mudanças inclui perda da sensação vibratória, do tato e da dor, assim como disfunção autonômica afetando a reatividade pupilar, a regulação da temperatura corporal e o controle vascular cardíaco e periférico (Freitas, 2018).

O ciclo sono-vigília se modifica com o envelhecimento. Os níveis de melatonina tanto diurnos quanto noturnos diminuem na maioria das pessoas, interferindo no sono, visto que este hormônio tem efeito hipnótico. As queixas de insônia, sonolência diurna, despertares durante a noite e sono pouco reparador são frequentes (Freitas, 2018).

No trato gastrointestinal ocorre modificação da acidez gástrica e da motilidade, alterando a absorção dos fármacos. O fluxo hepático também é reduzido, diminuindo a capacidade de metabolização dos medicamentos. As alterações hepáticas associadas à redução da taxa de filtração glomerular também afetam a excreção tanto de fármacos quanto de metabólitos (Oliveira et al. 2017).

A função renal começa a diminuir de maneira progressiva, chegando a sua metade aos 85 anos. Ainda, pela sua função excretora, o declínio da função renal pode levar a intoxicação medicamentosa, particularmente perigosa no idoso (Freitas, 2018). A diminuição dos hormônios tireoidianos, especialmente a conversão de T4 em T3, sugere uma ação protetora 
para o organismo contra o catabolismo, levando à diminuição da taxa do metabolismo basal e ao aumento progressivo do tecido adiposo corporal (Freitas, 2018).

\subsubsection{Adequação de Medicamentos}

O processo de envelhecimento leva a um progressivo comprometimento da reserva funcional de órgãos e sistemas, e uma das consequências dessa situação é a maior sensibilidade dos idosos ao efeito dos medicamentos (Tommaso et al., 2021). Diante dos riscos decorrentes do envelhecimento e das diversas comorbidades, esses pacientes administram frequentemente uma grande variedade de medicamentos, instituindo-se a polifarmácia, visto, portanto, a relevância de avaliar a complexidade do regime terapêutico em prescrições destinadas a idosos (Bricola et al. 2018). Por essas razões, os idosos estão mais sujeitos à ocorrência de iatrogenias e reações adversas, que constituem importantes causas de óbito nessa população (Tommaso et al., 2021).

Mais da metade das iatrogenias sofridas por pacientes idosos têm relação com a terapêutica. Dentro desse contexto, estudos mostram que a maioria (80 a 90\%) dos idosos utilizam pelo menos um medicamento e 30 a $40 \%$ fazem uso de polifarmácia. Esse padrão de consumo medicamentoso, associado às doenças e alterações próprias do envelhecimento, desencadeia constantemente efeitos colaterais e interações medicamentosas com graves consequências a pacientes nessa faixa etária (Freitas, 2018).

Nos últimos anos, o uso dos psicotrópicos por idosos tornou-se tema de discussão necessária no âmbito da farmacoepidemiologia. Esse grupo, no entanto, apresenta maior vulnerabilidade aos eventos adversos relacionados aos mesmos, em muitos casos, são considerados medicamentos inapropriados (Ramos et al., 2020). Diante do exposto, nota-se que as listas e/ou os critérios para detecção de medicamentos inapropriados são úteis tanto para detecção de seu uso quanto para a não prescrição desses fármacos (Freitas, 2018).

Um desses instrumentos é o critério de Beers que compilou uma lista de medicamentos considerados inapropriados para utilização em idosos (Oliveira et al. 2017). A lista PRISCUS, concebida primariamente para a farmacopeia alemã, procura ser mais ampla contendo medicamentos não citados nos critérios de Beers-Fick. Ambos os instrumentos de avaliação de medicamentos potencialmente inapropriados para idosos detectaram aproximadamente 21 medicamentos em comum, notadamente benzodiazepínicos, anti-hipertensivos, ergotamina e derivados, laxantes, antiarrítmicos, anti-inflamatórios e antidepressivos (Gorzoni et al. 2012).

Em função disso, há uma preocupação na literatura em relação a medicamentos considerados inadequados para os idosos, eventos adversos, polifarmácia, redundância terapêutica e potenciais interações medicamentosas. Esses fatores, quando combinados com a automedicação e prescrição inadequada, contribuem para o fracasso terapêutico e geram custos desnecessários (Freitas, 2018).

A avaliação da farmacoterapia em idosos, portanto, é um importante instrumento de avaliação da qualidade da atenção prestada a este grupo etário, sendo que esforços para aprimorar a seleção, a prescrição, a dispensação e a utilização de fármacos devem constituir prioridade nos programas de atenção ao idoso (Ribas et al. 2014).

\subsection{Benzodiazepínicos e o Uso por Idosos}

\subsubsection{Conceitos}

Segundo Oliveira et al. (2020), para o idoso, o benzodiazepínico constitui uma solução efetiva para o alívio do sofrimento mental decorrente de problemas de vida, da solidão e da falta de sono, tornando-se tão indispensável quanto o alimento. Os riscos envolvidos no uso do medicamento são minimizados e não há receio da dependência. 


\subsubsection{Epidemiologia}

Benzodiazepínicos frequentemente são utilizados para tratamento de insônia. Entretanto, nos consensos internacionais sobre tratamento de insônia, não são drogas de primeira escolha. Na Classificação Internacional dos Distúrbios do Sono o uso crônico dos benzodiazepínicos é referido como causa de insônia, juntamente com uso de estimulantes do sistema nervoso central (AMB, 2013).

A distribuição dos usuários de benzodiazepínicos e antidepressivos destaca a maior prevalência dos indivíduos com faixa etária situada em torno dos 41 a 60 anos. Porém, quando se avalia a situação exclusivamente dos benzodiazepínicos, evidencia-se que a prevalência se mantém elevada entre os usuários com faixa etária entre 61 e 70 anos (Netto et al. 2012).

Uma revisão sobre efetividade clínica, custo benefício e diretrizes sobre o uso de benzodiazepínicos em idosos concluiu que as evidências disponíveis sugerem maiores chances de eventos cognitivos e psicomotores adversos entre os usuários de benzodiazepínicos, tais como quedas e fraturas. Nenhum trabalho avaliou com precisão a segurança, eficácia clínica e custo-efetividade do uso de benzodiazepínicos no tratamento de ansiedade ou problemas de comportamento em idosos. Apesar disso, a prevalência do seu consumo nesse segmento etário mantém-se muito elevada - cerca de $30 \%$-, muitas vezes cronicamente, por muitos anos, sendo ainda maior entre idosos mais velhos (Alvarenga et al. 2015). Isso mostra que, apesar dos riscos associados ao uso prolongado de benzodiazepínicos, especialmente entre os idosos, seu uso permanece frequente (Oliveira et al. 2020).

Nos países desenvolvidos, apesar da adoção do critério de Beers, como sinalizador de prescrição médica, os achados mostraram que seu consumo foi alto, independente das condições de saúde mental dos idosos (Ramos et al. 2020). Evitar o uso de medicações inapropriadas é uma estratégia simples e efetiva para redução das reações adversas aos medicamentos. Os medicamentos com ação psicoativa representam o maior grupo de fármacos potencialmente inapropriados para idosos, com elevado potencial de efeitos colaterais e de risco de interações medicamentosas (Tommaso et al. 2021).

\subsubsection{Eventos adversos dos benzodiazepínicos em idosos}

Os benzodiazepínicos têm composição lipossolúvel, o que permite uma rápida absorção e distribuição para o sistema nervoso central e uma diminuição da metabolização hepática, sendo que os idosos possuem uma maior sensibilidade a esses efeitos (Oliveira et al. 2017). O uso em portadores de múltiplas morbidades traz preocupação quanto à possibilidade de interações medicamentosas (Firmino et al. 2011). Assim, deve fazer parte da prática clínica a revisão periódica dos medicamentos utilizados por idosos (Freitas, 2018).

Entre os principais agravos à saúde potencialmente desencadeados pelo uso de benzodiazepínicos destaca-se o somatório do efeito depressor central do benzodiazepínico e da hipotensão ortostática comum aos anti-hipertensivos, acentuando o risco de quedas entre os pacientes particularmente entre idosos (Ramos et al. 2020).

Esses medicamentos podem ocasionar sintomas como sonolência diurna, diminuição da coordenação motora, alteração da memória, tontura, zumbidos, delirium, quedas e fraturas, reação paradoxal, intoxicação, além do risco de dependência. O uso crônico em idosos parece aumentar o risco de demência, principalmente da doença de Alzheimer (Oliveira et al. 2017). Diversos estudos já apontaram distorções nas prescrições de benzodiazepínicos, como o tempo de tratamento prolongado, uso equivocado para o alívio de quadros inespecíficos, uso por idosos e outras indicações incompatíveis com o perfil farmacológico da classe (Firmino et al. 2011).

A meia-vida de eliminação dos benzodiazepínicos é prolongada principalmente em idosos e pacientes com doença hepática. Com relação aos idosos, isso se deve tanto à diminuição da quantidade de enzimas metabolizadoras como ao aumento no volume de distribuição, devido à proporção maior de gordura em relação ao peso corporal. Além disso, a diminuição do metabolismo cerebral nesses pacientes os torna mais sensíveis às drogas depressoras do sistema nervoso central (Silva, 2017). 
Revisões sistemáticas apontam evidências acerca do aumento do risco de quedas entre idosos que consomem esses medicamentos. Adicionalmente, esses agentes podem ter seus níveis séricos elevados, com consequente aumento da toxicidade, quando associados a outros medicamentos (interações medicamentosas), tendo em vista que são substratos da enzima do citocromo P450 (Ramos et al. 2020).

\subsubsection{Alternativas terapêuticas e uso correto dos benzodiazepínicos}

A dose média prescrita de benzodiazepínicos e antidepressivos aos idosos, identificados no estudo sofre alterações mínimas em relação à utilizada pelos pacientes adultos. Esta avaliação é importante, principalmente devido aos riscos apresentados por esses medicamentos em pacientes idosos (Netto et al. 2012).

Os benzodiazepínicos são sabidamente ineficazes para o tratamento da insônia crônica em longo prazo, pois levam a uma série de alterações da arquitetura do sono, especialmente no uso crônico, o que contraindica seu uso para este propósito (AMB, 2013). Os benzodiazepínicos proporcionam redução da latência do sono, aumento do tempo total do sono e diminuição dos despertares durante o sono. Porém, observam-se importantes efeitos negativos, como redução do estágio 3 (sono profundo), podendo ainda haver não apenas diminuição do tempo total do sono REM, mas também redução da densidade de movimentos oculares rápidos, além de seu uso causar dependência, abuso e tolerância com frequência (Souza et al. 2018).

A maior utilização de benzodiazepínicos em pacientes com faixa etária mais elevada apresenta-se inadequada, pois, sabe-se que esses fármacos, em especial aqueles de meia vida longa não são recomendados para idosos, já que produzem sedação prolongada aumentando o risco de quedas e, consequentemente, de fraturas do quadril. Em idosos, quando se opta pelo uso desse tipo de medicação, deve-se dar preferência aos benzodiazepínicos que são conjugados por glicuronidação (lorazepam, oxazepam e temazepam), pois a via hepática de conjugação não é afetada pelo envelhecimento e, portanto, a meiavida do fármaco não é aumentada (Bricola et al. 2018). Esses medicamentos são considerados benéficos ao serem administrados a curto prazo ao paciente. No entanto, artigos têm demonstrado aumento do risco de desenvolver Alzheimer em pacientes em uso prolongado de benzodiazepínicos (Souza et al. 2018).

Para uma prescrição adequada, é importante levar em consideração as alterações farmacocinéticas e farmacodinâmicas que ocorrem com o envelhecimento (Tommaso et at. 2021), podendo ser necessário o ajuste de doses ou a avaliação da adequação desse medicamento frente às mudanças nos padrões farmacológicos com o envelhecimento (Freitas, 2018). À luz dos riscos envolvidos no uso desses medicamentos por idosos, faz-se imprescindível a conscientização dos profissionais de saúde para redobrar os cuidados na prescrição de benzodiazepínicos e monitoramento dos seus usuários (Oliveira et al. 2020).

A prescrição deve ser avaliada sistematicamente pelo médico que assiste o paciente. A indicação precisa e o tempo de uso são objetos a serem avaliados como as implicações decorrentes de seu uso prolongado. Os efeitos colaterais podem ser evitados por meio do uso de doses baixas por tempo limitado. Desse modo, a indicação do uso do medicamento deve estar clara assim como a dose e o tempo de prescrição (AMB, 2013).

Em consonância com recentes diretrizes terapêuticas, outros medicamentos vêm substituindo os benzodiazepínicos na abordagem de transtornos de ansiedade, como os antidepressivos mais novos e seguros em geriatria (por exemplo, sertralina), e a instituição de medidas não farmacológicas no manejo de transtornos do sono e sintomas de insônia, como a terapia comportamental cognitiva, têm sido indicadas (Oliveira et al. 2020).

A prescrição adequada para idosos é um grande desafio para todo médico que atende essa população. O grande objetivo deve ser minimizar as interações medicamentosas e diminuir a ocorrência de reações adversas aos medicamentos (Tommaso et al. 2021). 


\subsubsection{Desmame do uso de benzodiazepínicos}

Dentre as questões que permeiam o uso inadequado de benzodiazepínicos o tratamento prolongado é um dos mais frequentemente relatados. Estudos mostram que os prescritores tendem a manter o medicamento se o paciente já o usa há muito tempo e não se conhecem exatamente as circunstancias que levaram à indicação anterior (Ramos et al. 2020).

Não se deve prescrever benzodiazepínicos por mais do que 8 semanas, pois uso de 12 semanas já se caracteriza como uso crônico, que deve ser evitado para que não exista o risco de dependência. Para a maioria dos pacientes, a redução gradual é o melhor método de retirada, num processo palatino que pode durar até 6 meses. Em casos selecionados, de abuso de grande quantidade de comprimidos ou de drogas de meia-vida curta, pode ser recomendável a substituição por benzodiazepínicos de meia-vida longa (AMB, 2013).

É importante lembrar a necessidade de se reavaliar as medicações em todas as consultas médicas, a fim de analisar se todas continuam apropriadas às fases atuais das doenças, levando-se em conta a expectativa de vida do indivíduo naquele momento e os objetivos daquela terapêutica, tomando novas decisões com base nessas informações (Tommaso et al. 2021).

\section{Discussão}

Desse modo, os periódicos e capítulos de livros analisados permitiram identificar aspectos em relação ao uso de benzodiazepínicos a longo prazo por idosos. Com a análise global dos trabalhos foi possível perceber que é consenso entre os autores a preocupação em abordar os efeitos dessa classe de medicamentos nos idosos, principalmente em decorrência das alterações fisiológicas inerentes ao envelhecimento que favorecem ainda mais o surgimento dos efeitos prejudiciais nessa faixa etária.

Em relação aos benzodiazepínicos, a literatura estudada foi bem clara quanto aos efeitos adversos e quanto a recomendação de que esses medicamentos sejam prescritos por um curto prazo, sendo assim inadequado o uso a longo prazo não só por idosos, mesmo essa faixa etária sendo a mais afetada. Golan et al. (2012) explicam que frequentemente tais drogas são utilizadas como ansiolítico e sedativo, mas que há potencial desenvolvimento de dependência com o seu uso, assim como a chance de desenvolver tolerância, necessitando de doses cada vez mais elevadas.

Por meio da análise dos artigos selecionados observou-se que por mais que, na grande maioria dos casos, os benzodiazepínicos sejam usados como indutor de sono, seu efeito a longo prazo aumenta problemas referentes ao ciclo sonovigília, como afirmado por Freitas et al (2018). Por exemplo, a Diretriz da AMP aponta que existe tolerância para o efeito desses medicamentos como promotor do sono. Além disso, o sono tende a ser menos reparador. Dessa forma, a análise das bibliografias mostrou que os benzodiazepínicos são ineficazes para o tratamento da insônia crônica, pois levam a uma série de alterações da arquitetura do sono, o que contraindica seu uso para este propósito.

Nessa linha, Ramos et al. (2020) e Oliveira et al. (2020) realizaram seus estudos mostrando os principais efeitos dos benzodiazepínicos de forma prolongada e em doses inadequadas para idosos. Dentre tais efeitos, os mais bem elucidados foram os efeitos de sonolência diurna, diminuição da coordenação motora, alteração da memória, tontura, zumbidos, delirium, quedas e fraturas, reação paradoxal, intoxicação, além do risco de dependência.

Foi importante analisar também o quanto o manejo dos fármacos é fundamental no cuidado na saúde do idoso. Ribas et al. (2014) ressaltam em seu artigo que essa faixa etária tende a usar mais fármacos e, assim como Ramos et al. (2020) e Freitas et al. (2018), o ator cita o fato de que os idosos apresentam particularidades farmacodinâmicas e farmacocinéticas devido alterações típicas do envelhecimento. Foi identificado que dentre as alterações fisiológicas da senescência, destaca-se a diminuição no metabolismo de certos fármacos, dentre eles os benzodiazepínicos que dependem diretamente do metabolismo hepático que se encontra reduzido, aumentando assim o seu tempo de ação. Outra propriedade trazida por Oliveira et al. (2017) 
é a propriedade lipossolúvel dessa classe de fármacos, aumentando sua absorção pelo sistema nervoso central, fato esse que corrobora com os efeitos centrais mais acentuados referidos na Diretriz vinculada pela AMB e também referido pelo autor Souza et al. (2018).

Além disso, foram abordados também nas publicações o fato de que o uso em grande amostra dos benzodiazepínicos por idosos pode estar ligado ao fato de, como mostrado pelas alterações inerentes ao envelhecimento trazidas por Freitas e também por Oliveira et al. (2017), nessa idade começarem a aparecer distúrbios relacionados ao sono, quadros depressivos e também ao surgimento em alguns casos de doenças neurodegenerativas.

O estudo de base populacional publicado com Alvarenga et al. (2015), mostrou que, os idosos entrevistados que faziam uso de benzodiazepínicos, utilizavam esses fármacos de forma qualificada como "padrão de uso crônico", visto que os períodos relatados variavam de no mínimo seis meses chegando até quarenta anos de tratamento. Além disso, esses autores apontam que o uso de benzodiazepínicos aumenta com o envelhecimento da população analisada.

Somado a isso, é importante destacar que o as publicações analisadas apontam para a alta prevalência do uso dos benzodiazepínicos em idosos. O estudo transversal de Firmino et al. (2011), por meio da análise da dispensa de medicamentos nas farmácias das Unidades Básicas de Saúde do Município de Coronel Fabrician, MG, mostrou que os indivíduos com idade de 60 anos ou mais apresentaram uma prevalência de consumo de Diazepam 1,4 vez maior do que os mais jovens.

Outro fator que deve ser reforçado é o fato de os benzodiazepínicos serem fármacos contidos na lista de medicamentos de uso inadequado para idosos por estar associado ao aumento das doses relativas, dos efeitos adversos e de quedas nessa população. O capítulo do exemplar escrito por Tommaso et al. (2021), traz a lista atualizada dos critérios de Beers, onde os benzodiazepínicos aparecem como medicações que a prescrição deve ser evitada, tendo esse fato uma forte recomendação segundo os critérios.

O estudo feito por Fegadolli et al. (2019), trouxe entrevistas com profissionais da saúde a fim de verificar o papel destes profissionais no uso de benzodiazepínicos na atenção primária. $\mathrm{O}$ estudo mostrou que a falta de conhecimento sobre o assunto tem papel fundamental no abuso de tais substâncias. Desse modo é importante que haja investimentos em mecanismos de empoderamento dos profissionais para identificar o uso não adequado de tais substância e sugerirem manejos diferentes evitando o uso prolongado desses fármacos.

\section{Conclusão}

Através do estudo das publicações selecionadas, conclui-se que o uso de fármacos benzodiazepínicos a longo prazo não oferece benefícios, especialmente quando empregados como sedativos na insônia crônica, devido seu efeito prejudicial no ciclo sono-vigília. Além disso, foi possível evidenciar que, os efeitos adversos desses fármacos são ainda mais prejudiciais nos idosos, principalmente por favorecer os eventos de queda, uma grande vilã nessa faixa etária, causando graves riscos relacionados as fraturas e também maior dependência do idoso.

É observável também, mediante o estudo apresentado, que existe uma vasta bibliografia acerca dos contrapontos dos benzodiazepínicos e seu emprego na saúde do idoso e acerca das medicações proscritas para essa faixa etária. Entretanto, ainda é muito alta a prevalência da prescrição de medicações que o uso não é recomendado em idosos, sendo uma delas a classe farmacológica abordada no presente estudo.

Sendo assim, fica claro a importância de buscar formas menos prejudiciais para sanar as queixas em relação a sono e ansiedade em longevos, como por exemplo medidas de higiene do sono. Outro ponto seria investir na capacitação dos profissionais de saúde, a fim de identificar medicamento com uso não recomendado para idosos e propor formas de otimizar as prescrições. Essas ações devem ocorrer principalmente nas unidades de atenção primária à saúde, visto que esses locais foram 
os mais utilizados nas pesquisas populacionais dos artigos analisados, que mostraram alta prevalência do uso de benzodiazepínicos por idosos a longo prazo.

\section{Referências}

Alvarenga, J. M., Filho, A. I. L., Giacomin, K. C., Uchoa, E. \& Firmo, J. O. A. (2015). Uso de benzodiazepínicos entre idosos: o alívio de “jogar água no fogo", não pensar e dormir. Revista Brasileira de Geriatria e Gerontologia, 18(2), 249-258.

Associação Médica Brasileira (AMB). (2013). Abuso e Dependência de Benzodiazepínicos. Associação Médica Brasileira São Carlos Do Pinhal, São Paulo.

Bricola, S. A. P. C., Garcia, V. S., Walber, T. \& Arruda, M. M. (2018). Medicamentos: terapêutica segura. Editora Manole.

Fegadolli, C., Varela, N. M. D. \& Carlini, E. L. A. (2019). Uso e abuso de benzodiazepínicos na atenção primária à saúde: práticas profissionais no Brasil e em Cuba. Cad. Saúde Pública, 35 (6), 1-13.

Fiorelli, K. \& Assini, F. L. (2017). A prescrição de benzodiazepínicos no Brasil: uma análise da literatura. Arquivos Brasileiros de Ciências da Saúde. 2017; 42(1):40-44.

Firmino, K. F., Abreu, M. H. N. G., Perini, E. \& Magalhães, S. M. S. (2011). Fatores associados ao uso de benzodiazepínicos no serviço municipal de saúde da cidade de Coronel Fabriciano, Minas Gerais, Brasil. Cad. Saúde Pública, Rio de Janeiro, 27(6):1223-1232.

Freitas, E. V. (2018). Tratado de Geriatria e Gerontologia. (4. ed.). Guanabara Koogan.

Golan, D. E., Armstrong, E. J., \& Armstrong, A. W. (2012). Principles of Pharmacology: The Pathophysiologic Basis of Drug Therapy. 3 ed. Philadelphia.

Gorzoni, M. L., Fabbri, R. M. \& Pires, S. L. (2012). Medicamentos potencialmente inapropriados para idosos. Rev Assoc Med Bras., $58(4)$ :442-446.

Katzung, B. G. \& Trevor, A. J. (2017). Farmacologia básica e clínica. (13. ed.). AMGH.

Leonardi, J. G., Azevedo, B. M. \& Oliveira, A. C. C. (2017). Benzodiazepínicos e seus efeitos no sistema nervoso central. Revista Saúde em Foco. 9. ed.

Netto, M. U. Q., Freitas, O. \& Pereira, L. R. L. (2012). Antidepressivos e Benzodiazepínicos: estudo sobre o uso racional entre usuários do SUS em Ribeirão Preto-SP. Revista de Ciências Farmacêuticas Básica Aplicada, 33(1):77-81

Oliveira, A. L. M. L., Nascimento, M. M. G., Costa, E. C., Firmo, J. O. A., Costa, M. F. L. \& Filho, A. I. L. (2020). Aumento da utilização de benzodiazepínicos entre idosos mais velhos: Projeto Bambuí. Revista Brasileira Epidemiologia, 23 (1), 4-10.

Oliveira, H. S. B., Sousa, J. R. P., Donis, A. C. G. \& Manso, M. E. G. (2017). Utilização dos critérios de Beers para avaliação das prescrições em idosos portadores de doenças crônicas vinculados a um plano de saúde. RBCEH, 14 (3), 242-251.

Ramos, T. B. (2020). Fatores associados ao uso de psicotrópicos por idosos residentes no Município de São Paulo. Revista Ciência \& Saúde Coletiva, 25(11):4351-4360.

Ribas, C. \& Oliveira, K. R. (2014). Perfil dos medicamentos prescritos para idosos em uma Unidade Básica de Saúde do município de Ijuí-RS. Revista Brasileira de Geriatria Gerontolologia, 17 (1).

Ribeiro, L. C. C., Alves, P. B. \& Meira, E. P. (2009). Percepção dos idosos sobre as alterações fisiológicas do envelhecimento. Revista Ciência Cuidado em Saúde, 8(2), 220-227.

Silva, P. (2017). Farmacologia. (8.ed.). Guanabara Koogan.

Souza, F. J. F. B., Lima, G. F, Giancarlo, F. L. \& Kristian, M. (2018). Avaliação do padrão de sono em insones usuários de benzodiazepínicos e análise da trazodona como medicação substitutiva. Jornal Brasileiro de Psiquiatria, 67(2):80-86.

Sociedade Beneficente Israelita Brasileira Albert Einstein (SBIBAE). (2019). Nota técnica para organização da rede de atenção à saúde com foco na atenção primária à saúde e na atenção ambulatorial especializada - saúde da pessoa idosa. São Paulo: Hospital Israelita Albert Einstein: Ministério da Saúde.

Stahl, S. M. (2013). Stahl's essential psychopharmacology neuroscientific basis and practical application. (4. ed.) Syndicate of the Press of the University of Cambridge, England.

Tommaso, A. B. G., Moraes, N. S., Cruz, E. C. \& Kairalla, M. C. (2021). Geriatria: guia prático. (2. ed.) Guanabara Koogan, 2021. 